\title{
A Collocation-Based Algorithm for Analyzing Bifurcations in Phase Locked Loops with Tanlock and Sawtooth Phase Detectors
}

\author{
Bassam A. Harb $\mathbb{D}^{D}$, Ahmad Al-Ajlouni, and Ali Eyadeh \\ Communication Engineering Department, Yarmouk University, Irbid, Jordan \\ Correspondence should be addressed to Bassam A. Harb; bharb@yu.edu.jo
}

Received 14 March 2018; Accepted 11 June 2018; Published 4 July 2018

Academic Editor: Yakov Strelniker

Copyright (C) 2018 Bassam A. Harb et al. This is an open access article distributed under the Creative Commons Attribution License, which permits unrestricted use, distribution, and reproduction in any medium, provided the original work is properly cited.

\begin{abstract}
Analysis of bifurcation of second-order analog phase locked loop (PLL) with tanlock and sawtooth phase detectors is investigated. Both qualitative and quantitative analyses are carried out. Qualitatively, the basin boundaries of the attractors were constructed by plotting the stable and the unstable manifolds of the system. The basin boundaries show that the PLL under consideration for certain loop parameters has a separatrix cycle which terminates the limit cycle (out-of-lock state) and the loop pulls-in. This behavior is known in literature as homoclinic bifurcation and the value of the bifurcation parameter where this process occurs is called the pull-in range. Quantitatively, we propose a collocation-based algorithm to compute the separatrix cycle and the pull-in range. The separatrix cycle is approximated by a finite set of harmonics $\mathrm{N}$ with unknown amplitudes and by utilizing the fact that this limit cycle bifurcates from a separatrix cycle, a system of nonlinear algebraic equations is derived. For given values of filter parameters and gain, the algorithm numerically solves for the unknown amplitude of the harmonics and the value of the pull-in range simultaneously by evaluating the system at the collocation points. Results demonstrate that phase locked loop with sawtooth phase detector characteristics has the wider pull-in range followed by tanlock and sinusoidal, respectively.
\end{abstract}

\section{Introduction}

Phase locked loop is an electronic device with a voltagecontrolled oscillator that is continuously adjusted to match the phase or frequency of a sinusoidal input signal. It is an essential part in many electronic circuits used for the demodulation of Amplitude Modulation (AM) and a BPSK (Binary Phase Shift Keying) signals. PLL is used as frequency synthesizers where a stable frequency at multiples of an input frequency can be obtained $[1,2]$. PLL is used in modern wireless communication systems for synchronization, clock synthesis, and jitter reduction. In Code Division Multiple Access (CDMA) direct sequence spread spectrum systems (DS-SS), PLL is used to ensure exact code phase timing estimate. Such timing is influenced by clock offsets and by Doppler [3]. Moreover, phase locked loop frequency synthesizers are used in the design of transceivers in wireless communication systems [4]. A classical PLL consists of three components: phase detector, low pass filter, and voltagecontrolled oscillator. Figure 1 shows the nonlinear model of the PLL under consideration where phase detector is modeled by the nonlinear function $g(\varphi)$.

The closed loop phase error $\varphi$ is given by $\varphi \equiv \omega_{\mathrm{i}} \mathrm{t}$ $\theta_{\mathrm{v}}$ where $\omega_{\mathrm{i}}$ is the frequency of the incoming signal and $\theta_{\mathrm{v}}$ is the phase of the VCO. Other voltage-controlled oscillator parameters are the VCO gain $\left(\mathrm{k}_{\mathrm{v}}\right)$ and the free running frequency $\left(\omega_{0}\right)$. The quantity $\omega_{\mathrm{os}}=\omega_{\mathrm{i}}-\omega_{\mathrm{o}}$ is known in the literature as the loop detuning frequency whereas the closed loop gain is denoted by G. The role of the loop is to match the phase of the signal out of the VCO with the phase of the input signal. It is known that the phase error which is the solution of the autonomous, nonlinear differential equation describing the closed loop phase error loop can be in two different states. One is called the equilibrium state (phase-lock) where the phase error is constant and asymptotically stable and the other one is called the out-of-lock state (false lock) where the phase error is a limit cycle (periodic solution) [5]. Of interest here is the behavior of this limit cycle and how does this limit cycle affect the pull-in process. For second-order PLL, previous results showed that a limit cycle exists as a result of 


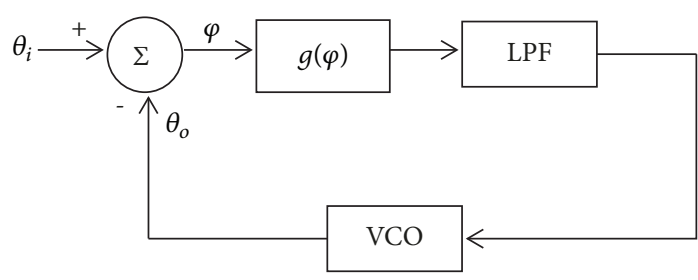

FIGURE 1: Nonlinear model of phase locked loop.

bifurcation from a separatrix cycle (homoclinic bifurcation). The value where bifurcation occurs $\left(\omega_{\mathrm{os}}=\omega_{\mathrm{p}}\right)$ is found to be the value of the pull-in range; i.e., for values of $\omega_{\mathrm{os}}<\omega_{\mathrm{p}}$, the loop pulls-in and the PLL is said to be phase locked, whereas for values of $\omega_{\text {os }}>\omega_{\mathrm{p}}$, a limit cycle bifurcates and in this case, the PLL is said to be false-locked. This behavior will be discussed analytically later in this paper. Many studies have been conducted and results concerning nonlinear analysis and bifurcations in phase locked loops can be found in the literature [6-8]. For example, the work by Stensby [9] and Harb and Stensby [10] showed that second-order phase locked loop undergoes saddle-node bifurcation. Stensby and Harb [11] proposed a numerical technique based on Galerkin procedures to compute the pull-in range of a second-order, Type I PLL with sinusoidal phase detector. Later Harb and Ajlouni [12] used a collocation-based algorithm for determining the pull-in range in second-order PLL with sinusoidal phase detector. Harb [13] proposed an iterative algorithm to solve for the pull-in range for different phase detector characteristics. Higher order phase locked loops have been studied in the literature as well. For example, Harb and Harb [14] studied third-order phase locked loop where the loop undergoes Hopf bifurcation and by using the multiple scale method they computed the limit cycle which was born as a result of this bifurcation. Monteiro et al. [15] computed the capture range of third-order PLL based on the location of the equilibrium points. Piquira et al. [16] used bifurcation theory in determining the lock-in range for third-order phase locked loops. The effect of time delay on the pull-in range for phase locked loop has been studied in the literature [17]. The work by Buckwalter and York [18] studied the effect of time delay on the pull-in range of high frequency phase locked loop with sinusoidal phase detector. Harb [19] studied the effect of time delay in PLL with tanlock and sawtooth phase detector characteristics. Stensby [20] derived an exact formula for computing the pull-out frequency for a second-order Type II PLL with triangular phase detector. The idea of synchronization in coupled phase locked loop was investigated by Buckwalter et al. [21] using harmonic balance technique.

Digital phase locked loop with tanlock phase detector characteristics has gained a great attention in literature but less effort has been paid to analog PLL with tanlock and sawtooth phase detectors [22-27]. In this paper, a collocationbased algorithm is used here to analyze bifurcations and computing the pull-in range of a second-order, Type II phase locked loop with tanlock and sawtooth phase detector characteristics. The effect of using different phase detector on the pull-in range will be evaluated and more attention will be paid to the qualitative analysis where the basins of attraction (equilibrium points and limit cycles) are identified. Moreover, the behavior of the attractors as one of the loop parameters changes will be analyzed and will be used in the formulation of the two parts of the algorithm. The first part approximates the limit cycle by a finite number of harmonics with unknown amplitudes. These amplitudes constitute $2 \mathrm{~N}+1$ unknowns that must be found. With frequency detuning, where the limit cycle exists as another unknown, the total number of unknowns will be $2 \mathrm{~N}+2$ to be determined. By evaluating the limit cycle at the collocation points, a set of $(2 \mathrm{~N}+1)$ algebraic equations is obtained. In order to solve for the unknowns simultaneously, one more equation is required which constitutes the other part of the algorithm and is obtained by evaluating the limit cycle at the saddle point. Given values of loop parameters and loop gain, the algorithm computes one period of the separatrix cycle and the value of detuning frequency $\left(\omega_{\text {os }}=\omega_{\mathrm{p}}\right)$ where the loop pulls-in.

The organization of the paper is as follows: Section 2 contains the mathematical model of the PLL under investigation. The qualitative behavior of the PLL is presented in Section 3. Section 4 discusses the proposed method and the simulation. Results and conclusions are presented in Section 5.

\section{Second-Order, Type II Phase Locked Loop Model}

In this section, the nonlinear differential equation that characterizes the dynamics of the PLL shown in Figure 1 is derived. The loop filter is given by the following transfer function:

$$
F(s)=\frac{s+a}{s+b}
$$

and the phase detector characteristics, $\mathrm{g}(\varphi)$, is periodic function with period of $2 \pi$. The general form of the closed loop phase error satisfies [10]

$$
\frac{\mathrm{d}^{2} \varphi}{\mathrm{dt}^{2}}+\left(\mathrm{b}+\mathrm{Gg}^{\prime}(\varphi)\right) \frac{\mathrm{d} \varphi}{\mathrm{dt}}+\mathrm{aGg}(\varphi)=\mathrm{b} \omega_{\mathrm{os}}
$$

where $\mathrm{G}$ is the closed loop gain and $\omega_{\mathrm{os}}$ is defined as the difference between the frequency of the incoming signal $\left(\infty_{i}\right)$ and the VCO free running frequency $\left(\omega_{0}\right)$. In this paper, a tanlock and sawtooth detectors are used. The general form of the tanlock phase detector is given by

$$
g(\varphi)=\frac{(1+\gamma) \sin \varphi}{1+\gamma \cos \varphi}
$$

A sinusoidal phase detector is obtained by letting $\gamma=0$, while, for $\gamma=0.816$, the phase detector becomes sawtooth.

\section{Qualitative Analysis of Second-Order Phase Locked Loop}

In this section, the behavior of the solution of the nonlinear differential equation is explained qualitatively. A PLL with sinusoidal phase detector characteristic is assumed here for 


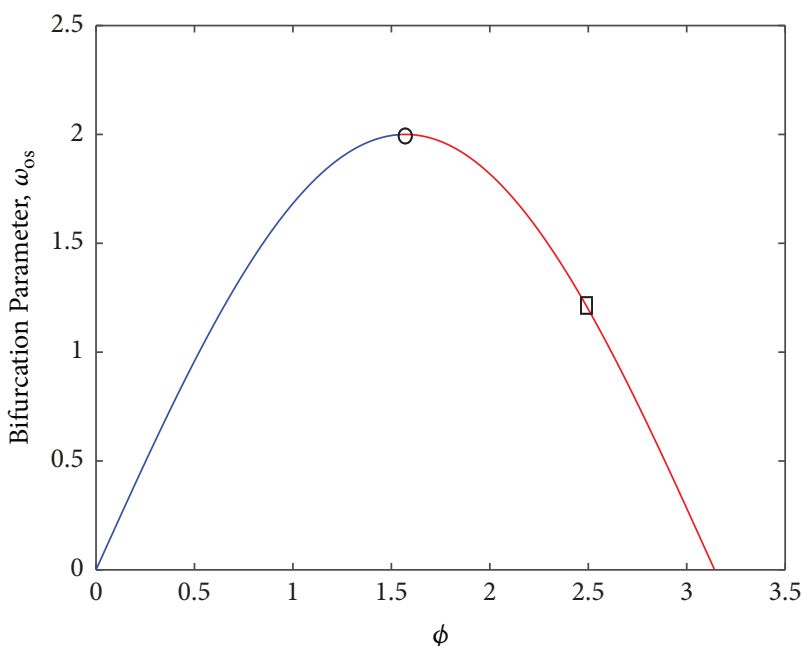

FIGURE 2: Bifurcation diagram for system (4).

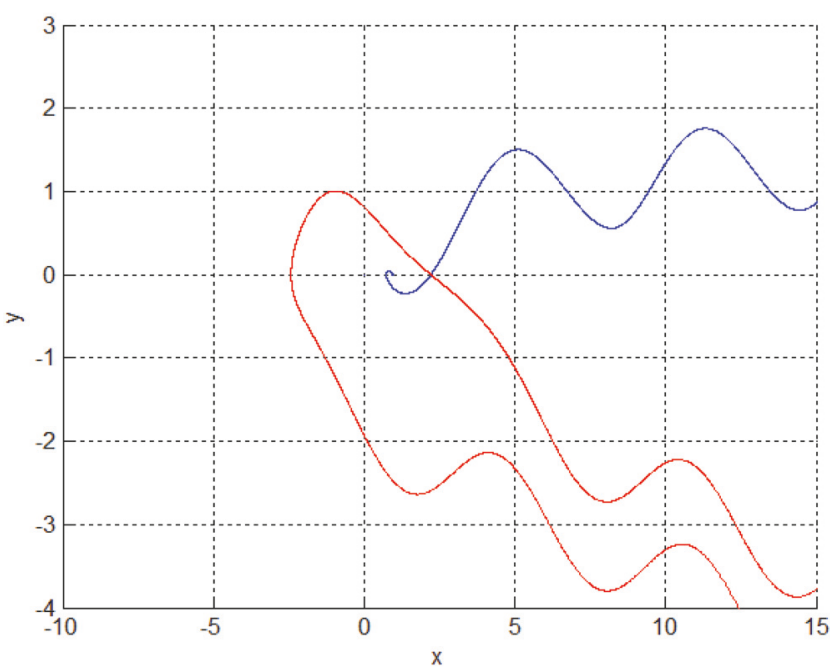

FIGURE 3: Stable and unstable manifolds of the system for $\omega_{\mathrm{os}}=1.8$.

the analysis. In this case, the nonlinear differential equation (2) becomes

$$
\frac{\mathrm{d}^{2} \varphi}{\mathrm{dt}^{2}}+(\mathrm{b}+\mathrm{G} \cos (\varphi)) \frac{\mathrm{d} \varphi}{\mathrm{dt}}+\mathrm{aG} \sin (\varphi)=\mathrm{b} \omega_{\mathrm{os}}
$$

The system has two equilibrium points which are given by

$$
\begin{aligned}
& \left(\varphi_{1}, \dot{\varphi}_{1}\right)=\left(\sin ^{-1}\left(\frac{\mathrm{b} \omega_{\mathrm{os}}}{\mathrm{aG}}\right), 0\right) \\
& \left(\varphi_{2}, \dot{\varphi}_{2}\right)=\left(\pi-\sin ^{-1}\left(\frac{\mathrm{b} \omega_{\mathrm{os}}}{\mathrm{aG}}\right), 0\right)
\end{aligned}
$$

With all parameters strictly positive, the set of equilibrium points given by (5a) are stable while those given by (5b) are unstable (saddle points). The bifurcation diagram for the system is shown in Figure 2.

In the figure, the $\mathrm{x}$-axis represents $\varphi$ and the $\mathrm{y}$-axis represents the parameter $\omega_{\text {os }}$. The diagram is plotted at fixed values

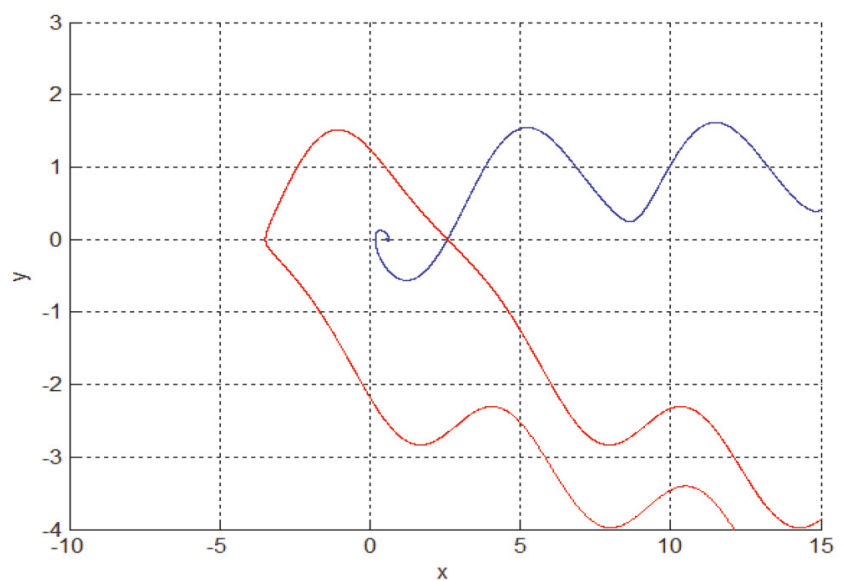

FIGURE 4: Stable and unstable manifolds of the system for $\omega_{\text {os }}=1.4$.

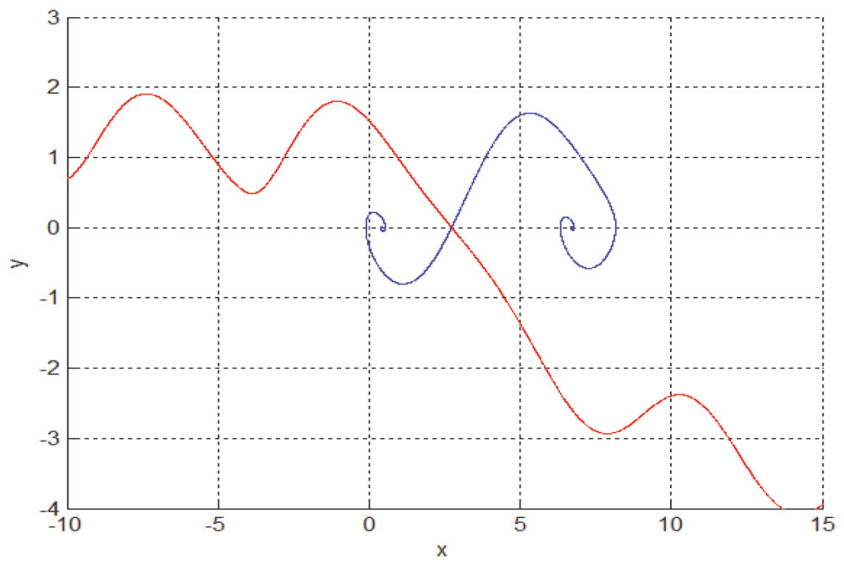

FIGURE 5: Stable and unstable manifolds of the system for $\omega_{\mathrm{os}}=1$.

of $\mathrm{b}=0.1 ; \mathrm{a}=0.4$; and $\mathrm{G}=0.5$ (the only varying parameter is $\left.\omega_{\text {os }}\right)$. The blue line stands for stable equilibrium point (knot or focus) where the red line stands for the saddle point. The empty circle stands for the saddle point bifurcation. This bifurcation occurs at the point $\omega_{\mathrm{os}}=\mathrm{aG} / \mathrm{b}=2$. Two equilibrium points occur after this bifurcation-the stable and the unstable (saddle) points. The square symbol stands for the homoclinic bifurcation (saddle-saddle connection). The homoclinic bifurcation damages the limit cycle-only equilibrium points exist in this system at the values of $\omega_{\text {os }}$ lower than the one corresponding to the ordinate of the square. Two different attractors coexist in the region between the circle and the square-a stable limit cycle and a stable equilibrium point. The basin boundaries of these attractors can be constructed by plotting the stable and the unstable manifolds of the system. To do that, all parameters of the system are fixed as before; the only variable parameter is $\omega_{\text {os }}$. Figures 3, 4, and 5 show the stable (blue) and the unstable (red) manifolds for different values of $\omega_{\text {os }}$. All initial conditions inside the red "tongue" (at $\omega_{\text {os }}=1.8$ and $\omega_{\text {os }}=1.4$ ) are attracted to the stable fixed point; all initial conditions outside the "tongue" are attracted to the stable limit cycle. The parameter value $\omega_{\mathrm{os}}=1$ represents the system state after the homoclinic (separatrix cycle) bifurcation; the limit cycle does 
not exist anymore; the only attractor is the stable equilibrium point. Of course, all manifolds are periodic by $2 \pi$. Note that $\mathrm{x}=\varphi$ and $\mathrm{y}=\mathrm{d} \varphi / \mathrm{dt}$ in the plots.

3.1. Equilibrium Points and Their Stability. In this section, the equilibrium points will be determined. Also, the type and stability of these points will be identified for tanlock phase detector given by (3). Substitute (3) into (2) to get

$$
\begin{aligned}
\frac{\mathrm{d}^{2} \varphi}{\mathrm{dt}^{2}} & +\left(\mathrm{b}+\mathrm{G}\left(\frac{(1+\gamma) \cos \varphi+\gamma(1+\gamma)}{(1+\gamma \cos \varphi)^{2}}\right)\right) \frac{\mathrm{d} \varphi}{\mathrm{dt}} \\
& +\mathrm{a}_{2} \mathrm{G}\left(\frac{(1+\gamma) \sin \varphi}{1+\gamma \cos \varphi}\right)=\mathrm{b} \omega_{\mathrm{os}}
\end{aligned}
$$

Define $x=\varphi, y=\dot{\varphi}$.

Then (6) can be written as

$$
\begin{aligned}
\dot{x}= & y \\
\dot{y}= & -\left(b+G\left(\frac{(1+\gamma) \cos x+\gamma(1+\gamma)}{(1+\gamma \cos x)^{2}}\right)\right) y \\
& -a G\left(\frac{(1+\gamma) \sin x}{1+\gamma \cos x}\right)+b \omega_{\text {os }}
\end{aligned}
$$

Of interest here are the points where the loop is in the phase locked state. This is found by letting $\dot{x}=\dot{y}=0$ in (7). By doing so, equilibrium points exist at [13]

$$
\begin{aligned}
& \mathrm{x}=(\pi \\
& \left.-\sin ^{-1}\left(\frac{2 c_{1}-\sqrt{4 c_{1}^{2}-4\left(c_{1}^{2}-c_{2}^{2}\right)\left(1+c_{2}^{2}\right)}}{2+2 c_{2}^{2}}\right)\right) \\
& -2 \pi \mathrm{k} \\
& \mathrm{y}=0 \text {, }
\end{aligned}
$$

where $c_{1}=b_{1} \omega_{p} /\left(a_{1}(1+\gamma)\right) ; c_{2}=\gamma c_{1}, \omega_{p}=\omega_{\text {os }} / G_{.}, b_{1}=b$ / $\mathrm{G}, \mathrm{a}_{1}=\mathrm{a} / \mathrm{G}$.

The stability of the equilibrium points must be determined. In order to do that, one needs to find the eigenvalues of the Jacobian matrix. The Jacobian matrix is given by

$$
J=\left[\begin{array}{cc}
0 & 1 \\
-G g^{\prime \prime}(x) y-a G g^{\prime}(x) & -\left(b+G g^{\prime}(x)\right)
\end{array}\right]
$$

where $I$ is the derivative with respect to $\mathrm{x}$. The eigenvalues are the roots of the following characteristic equation:

$$
\lambda^{2}+\left(\mathrm{b}+\mathrm{Gg}^{\prime}(\mathrm{x})\right) \lambda+\mathrm{Gg}^{\prime \prime}(\mathrm{x}) \mathrm{y}+\mathrm{aGg}^{\prime}(\mathrm{x})=0 .
$$

It is shown that values of $c_{1}$ satisfy the following inequality [10]:

$$
\begin{aligned}
& \left(\gamma^{2}-1\right)\left[1+\gamma^{2} c_{1}^{2}\right]<-c_{1}^{2}[[1 \\
& -2 \sqrt{1-\left[1-\gamma^{2}\right]\left[1+\gamma^{2} c_{1}^{2}\right]}+1 \\
& \left.\left.-\left(1-\gamma^{2}\right)\left(1+\gamma^{2} c_{1}^{2}\right)\right]\right]
\end{aligned}
$$

The eigenvalues will be of opposite signs which imply the existence of a saddle point. This saddle point plays a major role for computing the separatrix cycle (saddle-saddle connection) and hence the pull-in range.

\section{Computation of the Separatrix Cycle and the Pull-In Range}

In this section, the value of detuning $\omega_{\text {os }}$ for which a separatrix cycle exists is computed using the collocation method. We follow the same procedures described by Harb and AlAjlouni [12]. The computational process is as follows. Consider the nonlinear ordinary differential equation given by (7). This equation can be written as

$$
\begin{aligned}
\frac{d y}{d x}= & -\left(b+G\left(\frac{(1+\gamma) \cos x+(\gamma+1) \gamma}{(1+\gamma \cos x)^{2}}\right)\right) \\
& -\frac{a G((1+\gamma) \sin x /(1+\gamma \cos x))+b \omega_{o s}}{y}
\end{aligned}
$$

or

$$
\begin{aligned}
y \frac{d y}{d x} & +\left(b+G\left(\frac{(1+\gamma) \cos x+(\gamma+1) \gamma}{(1+\gamma \cos x)^{2}}\right)\right) y \\
& +a G\left(\frac{(1+\gamma) \sin x}{1+\gamma \cos x}\right)-b \omega_{o s}=0
\end{aligned}
$$

The periodic limit cycle of (13) can be represented by a finite number of harmonics and is given by

$$
y=\alpha_{0}+\sum_{k=1}^{N} \alpha_{k} \cos (k x)+\beta_{k} \sin (k x)
$$

where $\mathrm{N}$ is the number of harmonics. The main task is to solve for the amplitudes of the harmonics and the value of $\omega_{\mathrm{os}}$ where bifurcation occurs for given loop gain $(G)$. Note that the amplitude coefficients $\left(\alpha_{\mathrm{o}}, \alpha_{1}, \ldots, \alpha_{\mathrm{N}}, \beta_{1}, \ldots, \beta_{\mathrm{N}}\right)$ and $\omega_{\mathrm{os}}$ are unknowns to be determined. The first $(2 \mathrm{~N}+1)$ algebraic equations are obtained by defining the residual $\mathrm{R}$ to be

$$
\begin{aligned}
\mathrm{R}= & \mathrm{y} \frac{\mathrm{dy}}{\mathrm{dx}}+\left(\mathrm{b}+\mathrm{G}\left(\frac{(1+\gamma) \cos \mathrm{x}+(\gamma+1) \gamma}{(1+\gamma \cos \mathrm{x})^{2}}\right)\right) \mathrm{y} \\
& +\mathrm{aG}\left(\frac{(1+\gamma) \sin \mathrm{x}}{1+\gamma \cos \mathrm{x}}\right)-\mathrm{b} \omega_{\mathrm{os}}=0
\end{aligned}
$$


Next, we need to evaluate (15) at the collocation points which are the zeros of orthogonal polynomials such as Chebyshev polynomial or Legendre polynomial [28]. A Chebyshev polynomial of the first kind of degree $(2 \mathrm{~N}+2)$ is used which is given by

$$
\begin{aligned}
& \mathrm{T}_{0}(\mathrm{x})=1, \\
& \mathrm{~T}_{1}(\mathrm{x})=\mathrm{x}, \\
& \mathrm{T}_{\mathrm{n}}(\mathrm{x})=2 \mathrm{xT}_{\mathrm{n}}(\mathrm{x})-\mathrm{T}_{\mathrm{n}-1}(\mathrm{x})
\end{aligned}
$$

Solving for $R$ at the collocation points $x \mathrm{j}$ (zeros of the derivative of Chebyshev polynomial) yields $(2 \mathrm{~N}+1)$ algebraic equations with $(2 \mathrm{~N}+2)$ unknowns which are given by

$$
\begin{aligned}
\mathrm{R}\left(\mathrm{xj} ; \alpha_{0}, \alpha_{1}, \ldots, \alpha_{\mathrm{N}}, \beta_{1}, \ldots, \beta_{\mathrm{N}}, \omega_{0 \mathrm{~S}}\right)= & \\
& \text { for } 0 \leq \mathrm{j} \leq 2 \mathrm{~N}
\end{aligned}
$$

As mentioned before, another equation is required to solve for the $2 \mathrm{~N}+2$ unknowns simultaneously. This equation is obtained from the qualitative analysis discussed in Section 3 where it is verified that the separatrix cycle must approach the saddle point. Note that (7) has a saddle point for a single value of $\omega_{\text {os }}$ which is given by (8). This result leads to the $(2 N+2)$ th which is given by

$$
\begin{aligned}
& \mathrm{y}(\mathrm{x}) \mid \mathrm{x}_{\text {saddle }}=(\pi \\
& \left.-\sin ^{-1}\left(\frac{2 c_{1}-\sqrt{4 c_{1}^{2}-4\left(c_{1}^{2}-c_{2}^{2}\right)\left(1+c_{2}^{2}\right)}}{2+2 c_{2}^{2}}\right)\right) \\
& =0
\end{aligned}
$$

Now, (17) and (18) have $(2 \mathrm{~N}+2)$ unknowns and $(2 \mathrm{~N}+2)$ equations. Solving these unknowns required initial estimates for the unknowns and a value for the loop gain $(G)$. This is done by approximating the limit cycle with only DC component, $\alpha_{0}$, and first harmonics, $\alpha_{1}$ and $\beta_{1}$, and assuming other unknowns to be zeros. The following example illustrates the procedures.

Example 1. In what follows, a numerical example is presented to test the algorithm outlined above for several values of $\mathrm{N}$. In this example, the loop filter parameters $(\mathrm{a}=100, \mathrm{~b}=$ 10 ) with $\mathrm{N}=1$ are used. In this case, the separatrix cycle is approximated by

$$
\mathrm{y} \equiv \alpha_{0}+\alpha_{1} \cos (\mathrm{x})+\beta_{1} \sin (\mathrm{x})
$$

and $R$ becomes

$$
\begin{array}{r}
\mathrm{R}=\left[\alpha_{0}+\alpha_{1} \cos \left(\mathrm{x}_{\mathrm{j}}\right)+\beta_{1} \sin \left(\mathrm{x}_{\mathrm{j}}\right)\right] \\
\cdot\left(-\alpha_{1} \sin \left(\mathrm{x}_{\mathrm{j}}\right)+\beta_{1} \sin \left(\mathrm{x}_{\mathrm{j}}\right)\right)
\end{array}
$$

$$
\begin{aligned}
& +\left(\mathrm{b}+\mathrm{G}\left(\frac{(1+\gamma) \cos \mathrm{x}_{\mathrm{j}}+(\gamma+1) \gamma}{\left(1+\gamma \cos \mathrm{x}_{\mathrm{j}}\right)^{2}}\right)\right) \\
& \cdot\left[\alpha_{0}+\alpha_{1} \cos \left(\mathrm{x}_{\mathrm{j}}\right)+\beta_{1} \sin \left(\mathrm{x}_{\mathrm{j}}\right)\right] \\
& +\mathrm{aG}\left(\frac{(1+\gamma) \sin \mathrm{x}_{\mathrm{j}}}{1+\gamma \cos \mathrm{x}_{\mathrm{j}}}\right)-\mathrm{b} \omega_{\mathrm{os}}=0
\end{aligned}
$$

A Chebyshev polynomial of degree 4 is used here and is given by

$$
\mathrm{T}_{4}(\mathrm{x})=8 \mathrm{x}^{4}-8 \mathrm{x}^{2}+1, \quad \mathrm{x} \in[-1,1]
$$

After normalization of $\mathrm{T}_{4}(\mathrm{x})$ in the interval $\mathrm{x} \in[-\pi, \pi]$ (note that one period of the separatrix cycle is needed), (21) becomes

$$
\mathrm{G}_{4}(\mathrm{x})=\frac{8 \mathrm{x}^{4}}{\pi^{4}}-\frac{8 \mathrm{x}^{2}}{\pi^{2}}+1, \quad \mathrm{x} \in[-\pi, \pi] .
$$

The values of collocation points are given by the zeros of the derivative of $\mathrm{G}_{4}(\mathrm{x})$. These values are substituted into (17) to get the following three algebraic equations:

$$
\begin{array}{r}
\mathrm{R}\left(\mathrm{x}=\frac{\pi}{\sqrt{2}} ; \alpha_{\mathrm{o}}, \alpha_{1}, \beta_{1}, \omega_{\mathrm{os}}\right)=0, \\
\mathrm{R}\left(\mathrm{x}=-\frac{\pi}{\sqrt{2}} ; \alpha_{\mathrm{o}}, \alpha_{1}, \beta_{1}, \omega_{\mathrm{os}}\right)=0, \\
\mathrm{R}\left(\mathrm{x}=0 ; \alpha_{\mathrm{o}}, \alpha_{1}, \beta_{1}, \omega_{\mathrm{os}}\right)=0 .
\end{array}
$$

And the fourth equation is given by

$$
\mathrm{y}(\mathrm{x}) \mid \mathrm{x}_{\text {saddle }}=0
$$

where $\mathrm{x}_{\text {saddle }}$ is given by (8). To solve for the above system, MATLAB was used for this task. For a value of $\omega_{\text {os }}, a, b$, and gain $\mathrm{G}$, and using (23), initial guess is obtained for the coefficients $\left(\alpha_{o}, \alpha_{1}, \beta_{1}\right.$, and $\left.\omega_{\text {os }}\right)$. For a specified number of harmonics $(\mathrm{N})$, these estimated coefficients are supplied to the algorithm while other coefficients $\left(\alpha_{2}, \alpha_{3}, \ldots, \alpha_{N}, \beta_{2}, \ldots, \beta_{N}\right)$ assumed to be all zeros. Figures 6,7 , and 8 show the phase plane plots ( $\phi$ versus $\mathrm{d} \phi / \mathrm{dt}$ ) of one period of separatrix cycles computed for different values of gain $G$ with $N=$ 10 for tanlock, sawtooth, and sinusoidal phase detectors, respectively. Fixe values of $\mathrm{a}=100$ and $\mathrm{b}=10$ throughout the simulation. Figure 9 shows values of $\mathrm{G}$ (gain) and $\omega_{\mathrm{os}}$ (pull-in detuning frequency) where a separatrix cycle exists for all phase detectors. Each point on this means that the PLL has a separatrix cycle. As $\left(G, \omega_{\text {os }}\right)$ moves away from the graph, no separatrix cycle exists and the PLL may reach a stable equilibrium state (phase-lock) or out-of-lock state as discussed in Section 3 which means that the separatrix cycle is the boundary between the phase-lock and the out-of- lock states. 


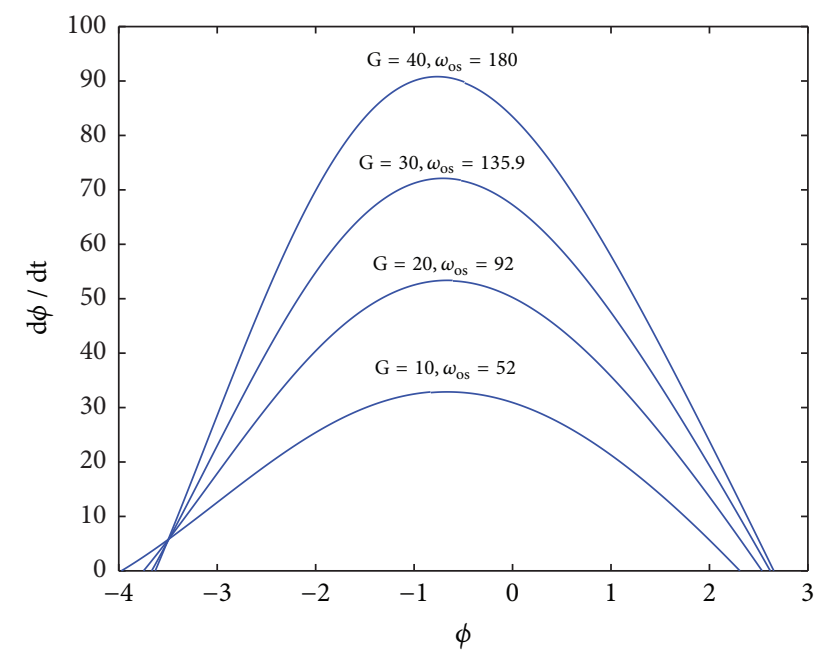

FIGURE 6: Separatrices for sinusoidal phase detector characteristics.

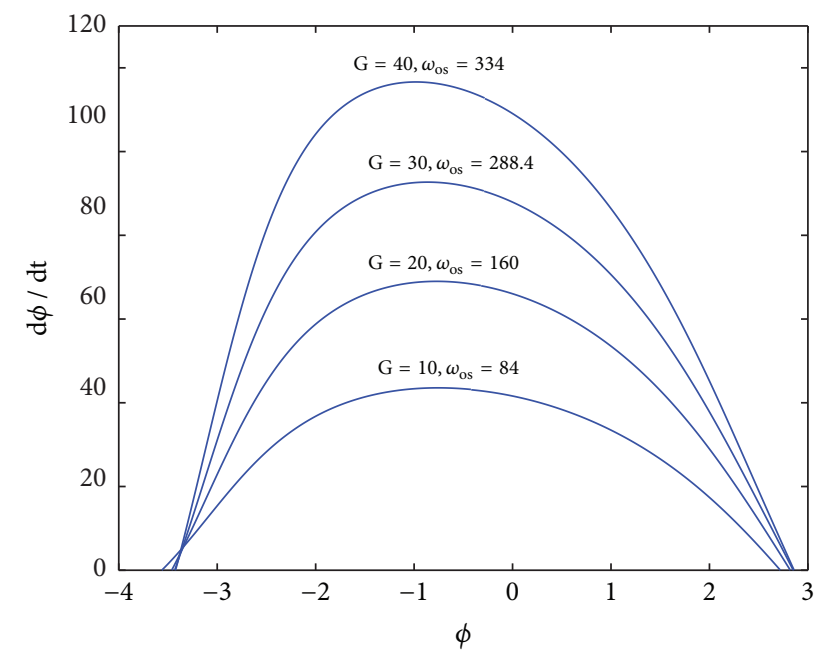

FIGURE 7: Separatrices for tanlock phase detector characteristics.

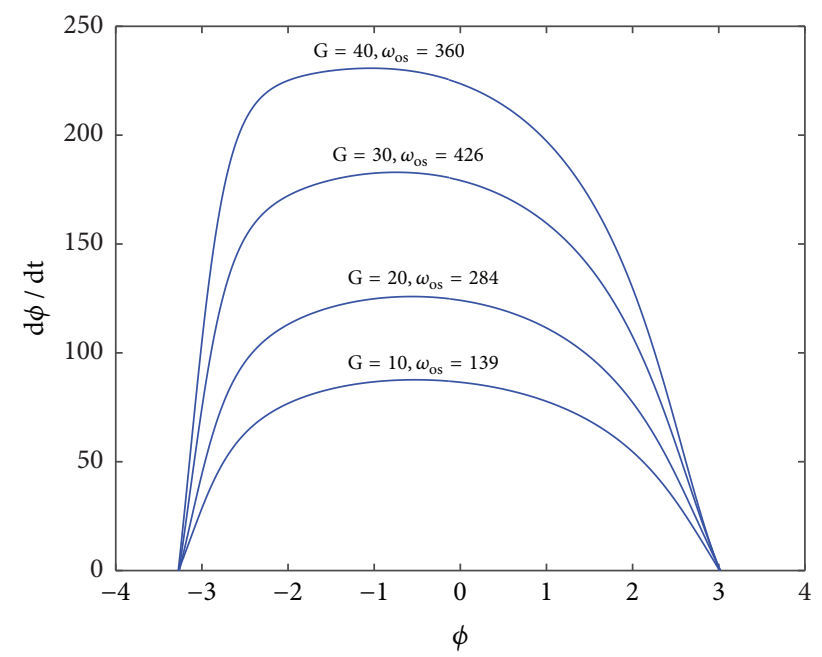

FIGURE 8: Separatrices for sawtooth phase detector characteristics.

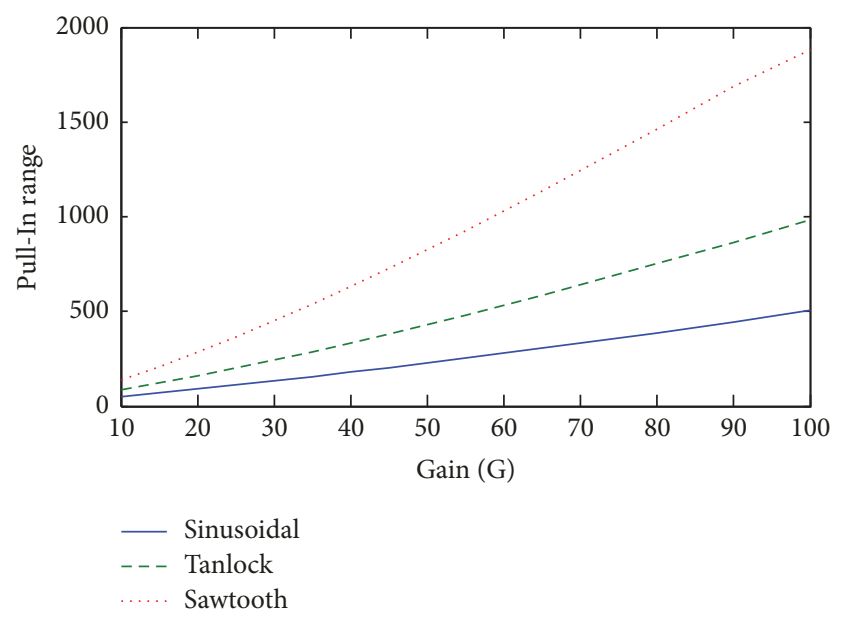

FIGURE 9: Pull-in range frequency versus closed loop gain for all phase detectors.

\section{Conclusions}

In this paper, new results on the nonlinear analysis of secondorder phase locked loop with tanlock and sawtooth phase detector characteristics are reported. We have proved the existence of the separatrix cycle qualitatively which have not been addressed in the literature. The basin boundaries of the attractors were constructed by plotting the stable and the unstable manifolds of the system. The qualitative analysis showed that the PLL pulls in as a result of homoclinic bifurcations. The value of the bifurcation parameter (pullin frequency) where this type of bifurcation occurs was computed via a collocation-based algorithm. Results showed that PLL with sawtooth phase detector characteristics has the wider pull-in range amongst all phase detectors considered in this study. These results should help system designer when evaluating other key performance parameters and the overall performance of the PLL.

\section{Data Availability}

The data used to support the findings of this study are included within the article.

\section{Conflicts of Interest}

The authors declare that they have no conflicts of interest.

\section{Acknowledgments}

The authors thank Minvydas Ragulskis, Professor of Applied Mathematics at Kaunas University of Technology of Lithuania, for his assistance in plotting the basin boundaries of the attractors.

\section{References}

[1] A. J. Viterbi, Principle of Coherent Communications, McGraw Hill, New York, NY, USA, 1966.

[2] A. Blanchard, Phase-Locked Loops, John Wiley, New York, NY, USA, 1976. 
[3] M. K. Simon, J. K. Omura, R. A. Scholtz, and B. K. Levitt, Spread Spectrum Communications, vol. 3, Computer Science Press, Rockville, MD, USA, 1985.

[4] H. Meyer and G. Aschied, Synchronization in Digital Communications, Wiley, New York, NY, USA, 1990.

[5] J. Stensby, "False lock and bifurcation in the phase locked loop," SIAM Journal on Applied Mathematics, vol. 47, no. 6, pp. 1177$1184,1987$.

[6] G. Leonov and N. Kuznetsov, Nonlinear Mathematical Models of Phase-Locked Loops. Stability and Oscillations, Cambridge Scientific Publisher, 2014.

[7] N. V. Kuznetsov, G. A. Leonov, M. V. Yuldashev, and R. V. Yuldashev, "Rigorous mathematical definitions of the hold-in and pull-in ranges for phase-locked loops," IFAC-PapersOnLine, vol. 48, no. 11, pp. 710-713, 2015.

[8] G. A. Leonov, N. V. Kuznetsov, M. V. Yuldashev, and R. V. Yuldashev, "Hold-in, pull-in, and lock-in ranges of PLL circuits: rigorous mathematical definitions and limitations of classical theory," IEEE Transactions on Circuits and Systems I: Regular Papers, vol. 62, no. 10, pp. 2454-2464, 2015.

[9] J. Stensby, "Saddle node bifurcation at a nonhyperbolic limit cycle in a phase locked loop," Journal of The Franklin Institute, vol. 330, no. 5, pp. 775-786, 1993.

[10] J. Stensby and B. Harb, "Computing the half-plane pull-in range of second-order PLLs," IEEE Electronics Letters, vol. 31, no. 11, pp. 845-846, 1995.

[11] B. Harb and J. Stensby, "The half-plane pull-in range of a second-order phase-locked loop," Journal of the Franklin Institute, vol. 333, no. 2, pp. 191-199, 1996.

[12] B. A. Harb and A. F. Al-Ajlouni, "A collocation-based algorithm for computing the pull-in range of a class of phase-locked loops," Nonlinear Dynamics, vol. 35, no. 3, pp. 249-258, 2004.

[13] B. Harb, "Computing the pull-in range of second-order phase locked loops with tanlock and sawtooth phase detectors," in Proceedings of the 11th International Conference on Circuits, Systems, Electronics, Control \& Signal Processing (CSECS'12), pp. 27-32, Montreux, Switzerland, 2012.

[14] B. A. Harb and A. M. Harb, "Chaos and bifurcation in a thirdorder phase locked loop," Chaos, Solitons \& Fractals, vol. 19, no. 3, pp. 667-672, 2004.

[15] L. H. A. Monteiro, D. N. F. Filho, and J. R. C. Piqueira, "Bifurcation analysis for third-order phase-locked loops," IEEE Signal Processing Letters, vol. 11, no. 5, pp. 494-496, 2004.

[16] J. R. C. Piqueira, "Using bifurcations in the determination of lock-in ranges for third-order phase-locked loops," Communications in Nonlinear Science and Numerical Simulation, vol. 14, no. 5, pp. 2328-2335, 2009.

[17] M. I. Schanz and A. X. Pelster, "Analytical and numerical investigation of the phaselocked loop with time delay," Journal of Chaos, Solitons and Fractals, vol. 11, pp. 142-148, 2005.

[18] J. Buckwalter and R. A. York, "Time delay considerations in high-frequency phase-locked loops," in Proceedings of the 2002 IEEE Radio Frequency Integrated Circuits (RFIC) Symposium, pp. 181-184, usa, June 2002.

[19] B. Harb, "Effect of time delay on the pull-in range of phase locked loops," Journal of Vibroengineering, vol. 16, no. 1, pp. 369377, 2014.

[20] A.-S. A. Huque and J. L. Stensby, "An exact formula for the pullout frequency of a 2nd-order type II phase lock loop," IEEE Communications Letters, vol. 15, no. 12, pp. 1384-1387, 2011.
[21] J. F. Buckwalter, T. H. Heath, and R. A. York, "Synchronization design of a coupled phase-locked loop," IEEE Transactions on Microwave Theory and Techniques, vol. 51, no. 3, pp. 952-960, 2003.

[22] B. C. Sarkar, S. S. De Sarkar, and T. Banerjee, "Control of bifurcation and chaos in a class of digital tanlock loops with modified loop structure," International Journal of Bifurcation and Chaos, vol. 24, no. 4, 2014.

[23] J. J. Uhran and J. C. Lindenlaub, "Experimental results for phase-lock loop systems having a modified nth-order tanlock phase detector," IEEE Transactions on Communication Technology, vol. 16, no. 6, pp. 787-795, 1968.

[24] A. H. Makarios and P. G. Farrell, "Noise and false-lock performance of the PSK-tanlock loop," IEEE Transactions on Communications, vol. 30, no. 10, pp. 2277-2284, 1982.

[25] R. C. Halgren, J. T. Harvey, and I. R. Peterson, "Improved acquisition in phase-locked loops with sawtooth phase detectors," IEEE Transactions on Communications, vol. 30, no. 10, pp. 2364$2375,1982$.

[26] Z. M. Hussain, B. Boashash, M. Hassan-Ali, and S. R. Al-Araji, "A time-delay digital tanlock loop," IEEE Transactions on Signal Processing, vol. 49, no. 8, pp. 1808-1815, 2001.

[27] J. C. Lee and C. K. Un, "Performance analysis of digital tanlock loop," IEEE Transactions on Communications, vol. 30, no. 10, pp. 2398-2411, 1982.

[28] U. M. Ascher, R. M. M. Mattheij, and R. D. Russell, Numerical solution of Boundary Value Problems for Ordinary Differential Equations, Prentice Hall Series in Computational Mathematics, Prentice Hall, Englewood Cliffs, NJ, USA, 1988. 


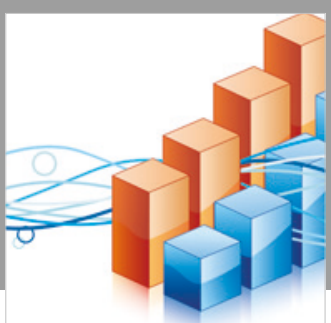

Advances in

Operations Research

\section{-n-m}
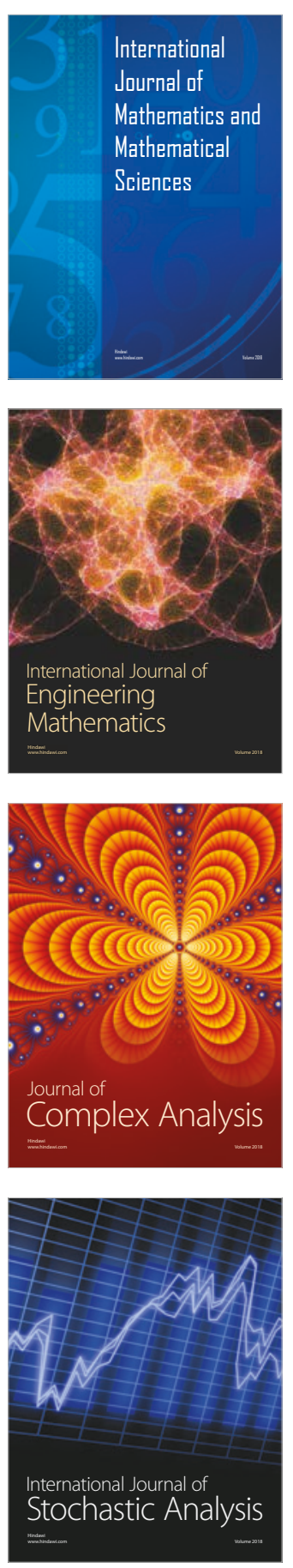
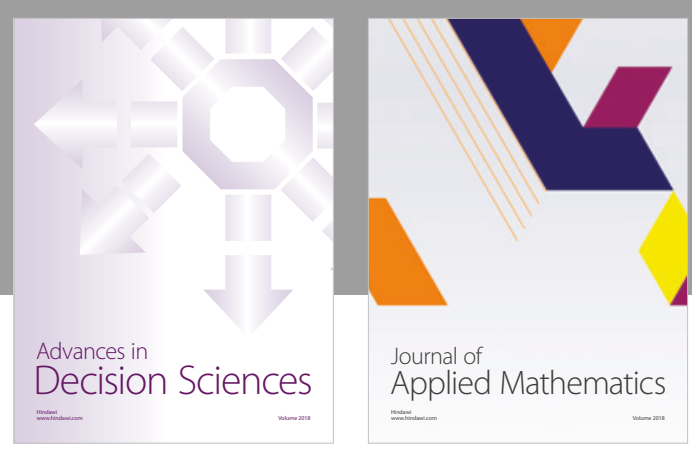

Journal of

Applied Mathematics
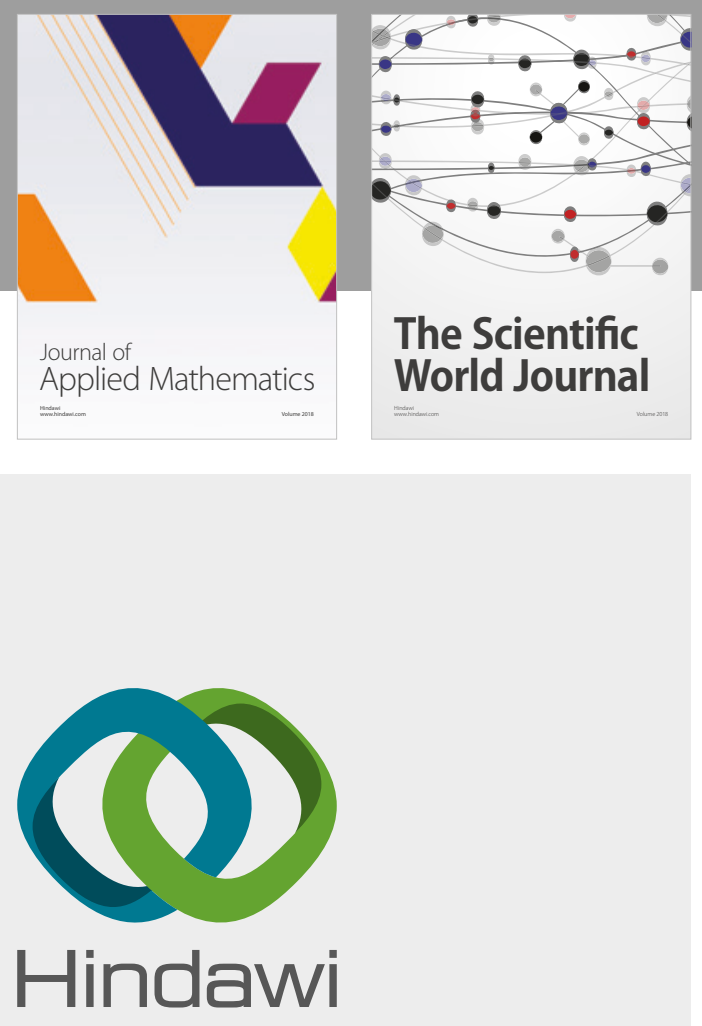

Submit your manuscripts at

www.hindawi.com

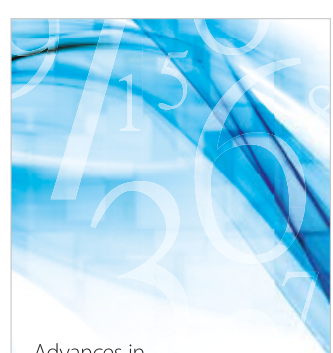

Advances in
Numerical Analysis
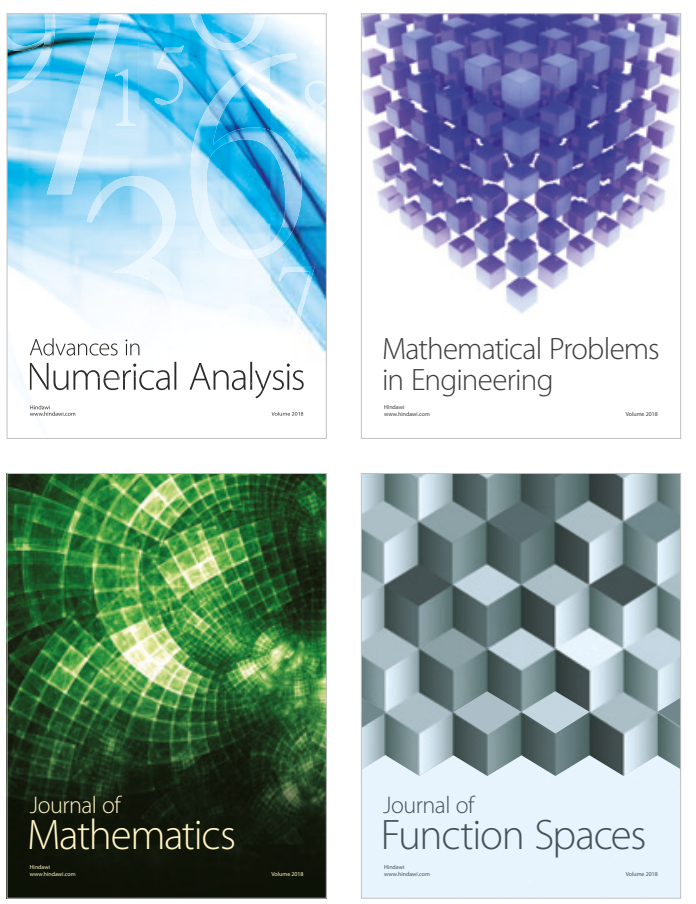

Mathematical Problems in Engineering

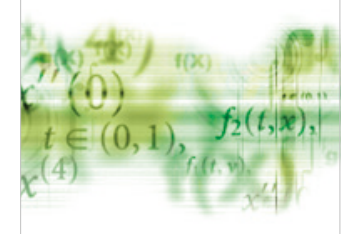

International Journal of

Differential Equations

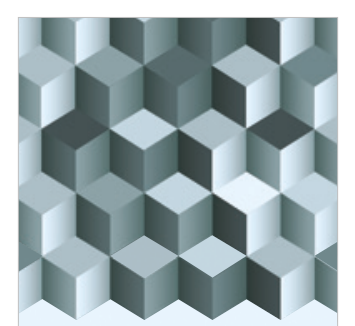

Journal of

Function Spaces

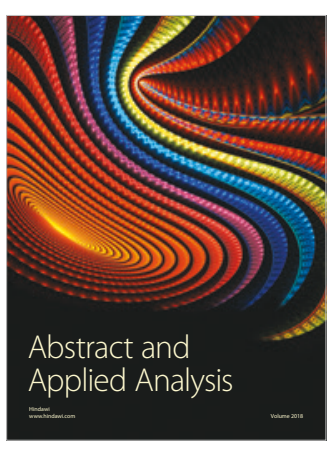

The Scientific

World Journal

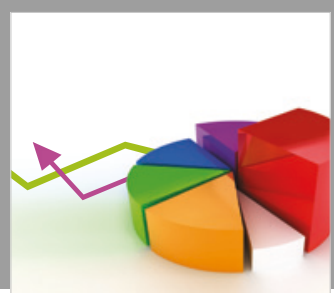

Journal of

Probability and Statistics
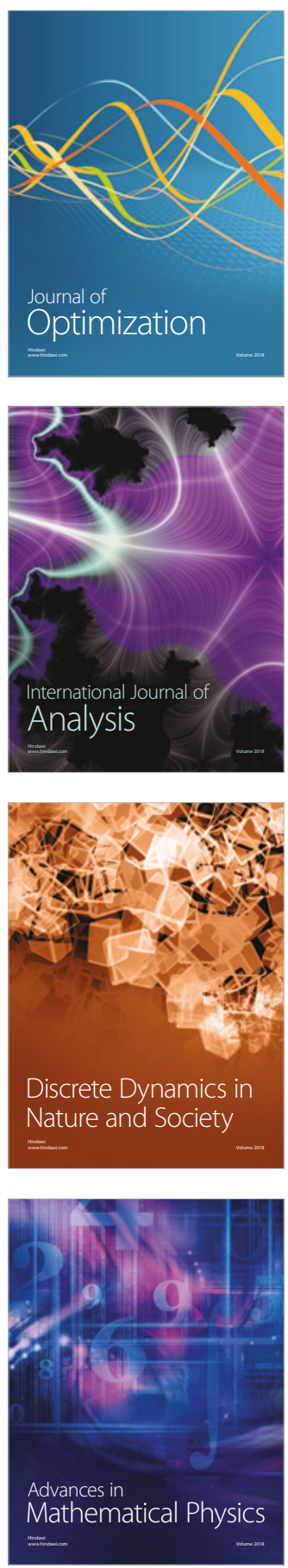